Ященко Ю.Б. ${ }^{1}$ Кондратюк Н.Ю. ${ }^{2}$

\author{
ОБОСНОВАНИЕ ПРОГРАММЫ РАННЕГО ВЫЯВЛЕНИЯ \\ И КОРРЕКЦИИ ФАКТОРОВ РИСКА РАЗВИТИЯ НЕИНФЕКЦИОННЫХ \\ ЗАБОЛЕВАНИЙ И ВНЕДРЕНИЕ СОСТАВЛЯЮЩИХ \\ ЭТОЙ ПРОГРАММЫ В ПОВСЕДНЕВНУЮ ПРАКТИКУ \\ МНОГОПРОФИЛЬНОГО УЧРЕЖДЕНИЯ
}

1) доктор медицинских наук, профессор, заведующий научным отделом организации медицинской помощи Государственного научного учреждения «Научно-практический центр профилактической и клинической медицины»

Государственного управления делами, ул. Верхняя, 5, г. Киев, Украина, 01014. e-mail: yayubor@yandex.ru

2) научный сотрудник научного отдела организации медицинской помощи Государственного научного учреждения «Научно-практический центр профилактической и клинической медицины» Государственного управления делами, ул. Верхняя, 5, г. Киев, Украина, 01014. e-mail: loriann2005@yandex.ru

Аннотация: В статье представлены результаты разработки комплекса составляющих программ раннего активного выявления неинфекционных заболеваний (НИЗ) и усовершенствования механизмов реализации процесса скрининга в практике врачей первичного звена. Представлена схема проведения профилактических медицинских осмотров и диспансерного наблюдения с использованием анамнестических скрининговых анкет.

Ключевые слова: профилактика; скрининг; неинфекционные заболевания; анамнестическое анкетирование; диспансеризация

Iashchenko Yu.B. ${ }^{1}$ Kondratiuk N.Yu. ${ }^{2}$

\section{JUSTIFICATION OF THE PROGRAM FOR EARLY DETECTION AND CORRECTION OF RISK FACTORS OF NONCOMMUNICABLE DISEASES AND IMPLEMENTATION OF COMPONENTS OF THIS PROGRAM IN THE DAILY PRACTICE OF MULTIDISCIPLINARY MEDICAL INSTITUTIONS}

1) MD, Professor, Head of Scientific Department of Medical Care, the Research and Practical Center for Preventive and Clinical Medicine. 5 Verkhnyaya St., Kiev, 01014, Ukraine. e-mail: yayubor@yandex.ru

2) Research Associate. Scientific Department of Medical Care, the Research and Practical Center for Preventive and Clinical Medicine. 5 Verkhnyaya St., Kiev, 01014, Ukraine. e-mail: loriann2005@yandex.ru

\begin{abstract}
The article presents the results of the development of a complex of components of the programs for early detection of active noncommunicable diseases (NCDs) and improving the mechanisms of implementation of the screening process in the practice of primary care physicians. The authors propose a scheme of preventive medical examinations and follow-up care with the use of anamnestic screening questionnaires.
\end{abstract}

Keywords: prevention; screening; non-communicable diseases; anamnestic questionnaire; clinical examination

Введение (актуальность). Развитие профилактического направления в здравоохранении является приоритетной задачей каждой страны, что отражено в стратегических документах международного, европейского и национального уровней. Весомым подтверждением этого является принятие в 2012 г. Новой Европейской политики «Здоровье 2020» и разработка в соответствии с ее основ национальных стратегий развития медицины.

Научно доказано, что профилактика является наиболее оптимальной стратегией сохранения здоровья населения. Профилактика неинфекционных заболеваний в 2,7 раза менее затратная, чем лечение больных. При этом здоровый образ жизни населения в 5 раз эффективнее, чем лечебно-диагностическая деятельность отрасли здравоохранения, связанная с сохранением и укреплением здоровья населения. Целенаправленное воздействие на управляемые факторы риска позволяет предупредить значительное число болезней, а своевременное и качественное лечение - их осложнения [1-5]. 

«Научный результат». Серия «Медицина и фармация». - Т.2, №1(7), 2016.

Международный национальный европейский и опыт применения профилактических программ и использования профилактических технологий свидетельствуют об их высокой медико-социальную и экономическую эффективность. В длительных проспективных исследованиях на представительных группах населения показана связь развития, прогрессирования и преждевременной смерти от основных неинфекционных заболеваний (НИЗ) с факторами риска. Концепция факторов риска стала методической основой исследований, посвященным вопросам профилактики НИЗ в развитых странах мира. Доказано, что проведение широкомасштабных мероприятий, направленных на профилактику и коррекцию факторов риска могут привести к существенному снижению смертности от основных НИЗ [6;7].

Ведущей частью профилактического направления здравоохранения и методом активного динамического наблюдения за состоянием здоровья всех групп населения является диспансеризация населения. Основная цель диспансеризации заключается в сохранении и укреплении здоровья населения, увеличении продолжительности жизни и повышении производительности труда путем активного выявления и лечения начальных форм заболеваний, изучение и устранение факторов, обусловливающих возникновение

и распространение заболеваний, а также проведение комплекса социальных, санитарногигиенических, профилактических, лечебнооздоровительных и организационных мероприятий [8-11].

Целью исследования является обоснование и разработка комплекса составляющих программ раннего активного выявления НИЗ и усовершенствования механизмов реализации процесса скрининга в практике врачей первичного звена. Исследование проводилось на базе многопрофильного учреждения Государственное научное учреждение «Научнопрактический центр профилактической и клинической медицины» Государственного управления делами.

Основными задачами исследования стали:

1. Анализ с точки зрения медико-социальной значимости, особенности организации профилактической работы на примере многопрофильного учреждения здравоохранения.

2. Разработка для медицинских работников первичного звена индивидуальной методики работы с конкретной популяцией по информации (о возможном вреде и риск и о преимуществах скрининга) по вопросу скрининга приемлемой в конкретном социуме и культуре для улучшения восприятия скрининга (на примере многопрофильного учреждения здравоохранения).

Использованные методы исследования: системного подхода, библиосемантичный, контент-анализ, ретроспективный анализ.

Результаты исследований и их обсуждение. На сегодня в Украине на улучшение состояния здоровья населения уже направлены ряд мероприятий профилактического направления.

Одним из таких мероприятий является планирование и проведение научных исследований по профилактике наиболее распространенных неинфекционных заболеваний.

В Украине существует положительный опыт по профилактике хронических неинфекционных заболеваний. Анализ результатов многоцентровых профилактических исследований, проведенных в 1988-1992 гг. двумя специализироваными научными центрами - НИИ кардиологии им. Н.Д. Стражеско (г. Киев) и НИИ терапии (г. Харьков), показал существенное влияние профилактических мероприятий на показатели здоровья населения. Благодаря их применению были снижены показатели смертности на 20-21\%, в основном, за счет уменьшения смертности от сердечнососудистых заболеваний более чем на $30 \%$ и сокращению распространенности артериальной гипертензии. Но, разработанные эффективные технологии первичной и вторичной профилактики основных НИЗ в Украине не получили широкого внедрения в практическом здравоохранении и надлежащего управленческого сопровождения по их применению.

Необходимость приоритетного развития профилактики указывается в ряде нормативноправовых, стратегических и программных документов. Провозглашение профилактики основных хронических неинфекционных заболеваний в качестве стратегического направления развития национальной системы здравоохранения требует научного обоснования и разработки современных стратегий, форм и методов ее осуществления.

Особое значение в решении данного вопроса должны скрининговые программы. Скрининг направлен на выявление предраковых состояний у категорий лиц с отсутствием каких-либо 

«Научный результат». Серия «Медицина и фармация». - Т.2, №1(7), 2016.

признаков или проявлений заболевания. Поскольку в Украине в настоящее время отсутствуют скрининговые программы и базовая методология скрининга, поэтому основным текущим задачам настоящего должно быть разработку усовершенствованной организационно-правовой модели скрининга наиболее распространенных НИЗ на примере многопрофильного лечебного учреждения.

Внедрение разработанных рекомендаций для осуществления превентивных мер для наиболее типичных состояний и заболеваний на основе скрининговых исследований позволит сократить ресурсные затраты, улучшить раннее выявление заболеваний и способствовать контролю за их ходом.

В ГНУ «НПЦ ПКМ» ГУД удалось сохранить диспансерный метод, как одно из величайших достижений в профилактической деятельности постсоветского здравоохранения. Почти все больные с хронической патологией находятся под диспансерным наблюдением у терапевтов и профильных специалистов. Охват пациентов с хронической патологией диспансерным наблюдением составляет около 90\%. Это показатель значительно выше, чем в г. Киеве и Украине.
Проведение профилактических осмотров прикрепленного населения осуществляется согласно утвержденным планам и графикам. Внедрение электронного реестра пациентов, совершенствование методики планирования профилактических осмотров прикрепленного контингента, проведение еженедельного контроля за организацией и результатами профилактической деятельности позволило достичь желаемого уровня охвата населения профилактическими осмотрами (более 90\%). В 2014 году план проведения профилактических осмотров выполнен на 99,1\%.

В структуре диспансерной группы населения трудоспособного возраста первые ранговые места занимают болезни органов пищеварения, системы кровообращения, эндокринной системы, новообразования и болезни мочеполовой системы. В течение 2012-2014 гг. среди населения трудоспособного возраста структура диспансерной группы стабильна. За трехлетний период отмечается увеличение доли населения трудоспособного возраста, которое находится под диспансерным наблюдением в результате болезней эндокринной системы (на 4\%) и уменьшения пациентов, страдающих болезнями системы кровообращения (на 8,6\%).

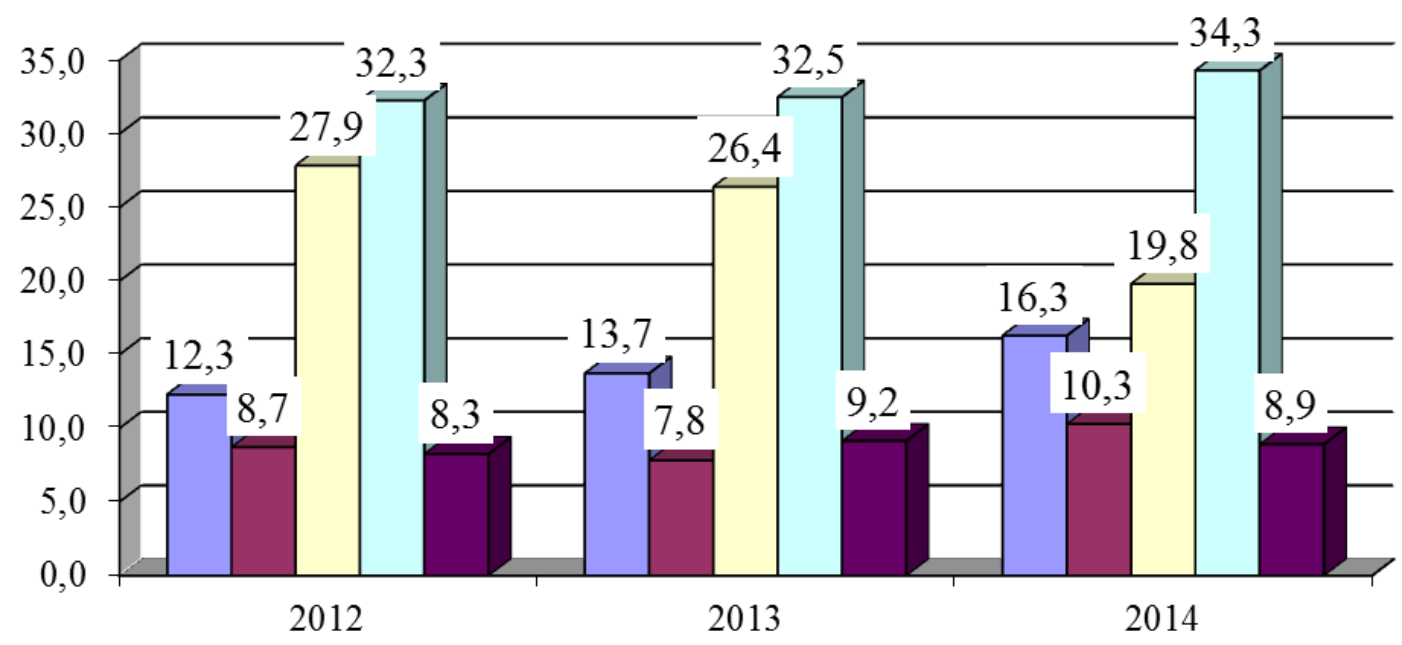

口болезни эндокринной системы 口болезни системы кровообращения 口новообразования 口болезни органов пищеварения

пболезни мочеполовой системы

Puc. 1. Динамика структуры основных классов болезней по каким ведется диспансерное наблюдение взрослого населения в ГНУ «НПЦ ПКМ» ГУС, 2012-2014 гг.

Fig. 1. Dynamics of the structure of the main classes of diseases whose medical check-up in the adult population is carried out in the SIS «RPC PCM» SAD 2012-2014.

Одним из самых весомых показателей эффективности диспансерного наблюдения является снятие с диспансерного наблюдения за счет выздоровления. Среди пациентов, 
находящихся под наблюдением, ежегодно выздоравливают около 2,3\% человек, еще в $15 \%$ случаев отмечается улучшение состояния здоровья. В то же время при оценке эффективности диспансеризации необходимо учитывать, что более $50 \%$ прикрепленных составляют лица пенсионного возраста. Поэтому, показатели эффективности диспансеризации отражают тот факт, что подавляющее большинство «Д» группы составляют лица пожилого и старческого возраста с тяжелыми хроническими заболеваниями, в которых практически не бывает полного выздоровления.

Среди болезней системы кровообращения большую долю занимает гипертоническая болезнь. Так, среди болезней системы кровообращения, по поводу взрослое население трудоспособного возраста находилось под диспансерным наблюдением, удельный вес гипертонической болезни составлял в 2012 году $57,4 \%$ в $2013-61,4 \%$, в $2014-91,4 \%$.

Для повышения качества проведения ежегодных профилактических медицинских осмотров и диспансерного наблюдения прикрепленного населения и четкого соблюдения маршрута пациента предложено условное деление таких осмотров на основные два этапа.

Цель первого этапа прохождения профилактического осмотра - это скрининг, то есть выявление признаков хронических неинфекционных заболеваний, факторов риска их развития, определения показаний к проведению дополнительных обследований и осмотров врачами-специалистами для уточнения диагноза заболевания (патологического состояния) на втором этапе диспансеризации.

Алгоритм проведения анамнестического анкетирования на уровне участкового врача (терапевта или семейного доктора):

1. Определение групп здоровья и распределение по следующим группам прикрепленного контингента.

2. Инструктаж обслуживаемого контингента, который пришел на профилактический осмотр, о порядке его прохождения, объем и последовательность проведения обследований с использованием плана диспансерного наблюдения.

3. Анамнестическое анкетирование для выявления неинфекционных заболеваний, факторов риска их развития (приложение 1).

4. Проведение обязательного минимума обследований (для I и II групп здоровья, для III группы + см. План диспансерного наблюдения).

Обязательный минимум диагностических исследований для пациентов, относящихся к I и II групा здоровья

Таблица 1 Table 1

Mandatory minimum diagnostic tests for patients belonging to I and II health groups

\begin{tabular}{|c|c|c|c|}
\hline \multicolumn{2}{|c|}{ Мужчины } & \multicolumn{2}{|c|}{ Женщины } \\
\hline Терапевт & АД & Терапевт & АД \\
\hline & OT & & OT \\
\hline & ИМТ & & ИМТ \\
\hline Хирург & & Хирург & \\
\hline \multicolumn{2}{|c|}{ Офтальмолог 1 раз в 2 года } & \multicolumn{2}{|l|}{ года } \\
\hline Исследования: & & Исследования: & \\
\hline & ЭКГ & & ЭКГ \\
\hline & УЗИ ЩЖ 1раз в 2 года & & УЗИ ЩЖ 1раз в 2 года \\
\hline & OAK & & OAK \\
\hline & $\mathrm{OAC}$ & & $\mathrm{OAC}$ \\
\hline & Глюкоза крови & & Глюкоза крови \\
\hline & ФГ 1 раз в 2 года & & ФГ 1 раз в 2 года \\
\hline Уролог & & $\begin{array}{l}\text { Гинеколог (включая взятие } \\
\text { мазков) }\end{array}$ & \\
\hline
\end{tabular}

5. Вторая (более) консультация (после получения данных обязательных обследований) и направления пациентов на дополнительное обследование и углубленное профилактическое консультирование на второй этап диспансеризации (! При необходимости!). 
Схема для определения рисков развития неинфекционных заболеваний по результатам анамнестического опроса (анкетирования)

Table 2

The scheme for determining the risks of non-communicable diseases based on the results of an anamnestic survey (questionnaire)

\begin{tabular}{|c|c|c|}
\hline Номера вопросов & $\begin{array}{l}\text { Оцениваемый вариант } \\
\text { ответа }\end{array}$ & РИСКИ \\
\hline 1-6- заболевание в анамнезе & ДА & Тактика и риск определяется терапевтом \\
\hline 7-10 - заболевание в семейном анамнезе & $\begin{array}{c}\text { ДА } \\
\text { НЕ ЗНАЮ }\end{array}$ & Тактика и риск определяется терапевтом \\
\hline $\begin{array}{c}\text { 11(МУЖЧИНЫ) - выявление подозрения } \\
\text { патологию предстательной железы }\end{array}$ & ДА & УРОЛОГИЧЕСКИЙ \\
\hline $\begin{array}{c}11 \text { (ЖЕНЩИНЫ) - выявление подозрения } \\
\text { патологии молочной железы }\end{array}$ & ДА & ОНКОЛОГИЧЕКИЙ \\
\hline 12 - выявление стенокардии & ДА & Тактика и риск определяется терапевтом \\
\hline $\begin{array}{c}13-14 \text { - выявление подозрения на } \\
\text { туберкулез, ХОБЛ или новообразование } \\
\text { легких }\end{array}$ & ДА & Тактика и риск определяется терапевтом \\
\hline 15 - выявление колебаний массы тела & ДА & Тактика и риск определяется терапевтом \\
\hline $\begin{array}{c}16 \text { - выявление показаний для консультации } \\
\text { проктолога }\end{array}$ & ДА & Консультация проктолога \\
\hline $17-$ выявление курения & ДА & $\begin{array}{l}\text { КАРДИОЛОГИЧЕСКИЙ } \\
\text { ОНКОЛОГИЧЕСКИЙ }\end{array}$ \\
\hline $\begin{array}{c}18 \text { - выявление низкой физической } \\
\text { активности }\end{array}$ & ДА & $\begin{array}{l}\text { КАРДИОЛОГИЧЕСКИЙ } \\
\text { ЭНДОКРИННЫЙ }\end{array}$ \\
\hline $\begin{array}{c}\text { 19-22 - выявление нерационального } \\
\text { питания }\end{array}$ & ДА & $\begin{array}{l}\text { КАРДИОЛОГИЧЕСКИЙ } \\
\text { НЕВРОЛОГИЧЕСКИЙ } \\
\text { ЭНДОКРИННЫЙ }\end{array}$ \\
\hline $\begin{array}{c}\text { 23-24-выявление подозрения наличия } \\
\text { эндокринных нарушений }\end{array}$ & ДА & ЭНДОКРИННЫЙ \\
\hline $\begin{array}{l}\text { 25-26 - выявление предвестников или } \\
\text { наличия депрессивных расстройств }\end{array}$ & ДА & ПСИХОЛОГИЧЕСКИЙ \\
\hline
\end{tabular}

Второй этап профилактического осмотра проводится с целью дополнительного обследования и уточнения диагноза заболевания, проведения углубленного профилактического консультирования.

\section{Заключение.}

Проведение профилактических осмотров прикрепленного населения осуществляется согласно утвержденным планам и графикам. Внедрение электронного реестра пациентов, совершенствование методики планирования профилактических осмотров прикрепленного контингента, проведение еженедельного контроля за организацией и результатами профилактической деятельности позволило достичь желаемого уровня охвата населения профилактическими осмотрами (более 90\%). В 2014 г. План проведения профилактических осмотров выполнен на 99,1\%.

Предложенный подход с обязательным проведением анамнестического опроса пациентов и дифференцированного подхода к назначению дополнительных методов исследования и консультации специалистов вторичного звена оказания медицинской помощи позволит обеспечить четкое соблюдение маршрута пациентов и сократить финансовые затраты для проведения ежегодных профилактических медицинских осмотров. Кроме этого проведение ежегодных профилактических медицинских осмотров, в дальнейшем, будет направлено на выявление факторов риска развития НИЗ и их коррекцию.

\section{Литература}

1. Блум Дэйвид И. Состояние здравоохранения в мировом масштабе // Мировое здравоохранение. 2014. №12. C. 6-11.

2. Глобальный план борьбы с диабетом 20112021. Международная Федерация диабета. Брюссель, 2010. C.7.

3. Мониторинг факторов риска неинфекционных заболеваний. Принцип поэтапной реализации, 

«Научный результат». Серия «Медицина и фармация». - Т.2, №1(7), 2016.

предложенный ВОЗ. Краткий обзор / Р. Бонита, М. де Куэртен,Т. Дуайер и др. - Женева: ВОЗ, 2001.

4. Kontis V., Mathers C.D., Rehm J.Contribution of six risk factors to achieving the $25 \times 25$ non-communicable disease mortality reduction target: a modelling study // The Lancet. 2014. Vol. 384. Pp. 427-437.

5. Di Cesare M., Bennett. J.E., Best N. The contributions of risk factor trends to cardiometabolic mortality decline in 26 industrialized countries // International Journal of Epidemiology. 2013. № 42. P. 838-848.

6. Ezzati M. Riboli E. Behavioral and dietary risk factors for non-communicable diseases // New England Journal of Medicine: Research \& Review. 2013. Vol. 369. Pp. 954-964.

7. Ezzati M., Riboli E. Can non-communicable diseases be prevented? Lessons from studies of populations and individuals // Science. 2012. Vol. 337. P. 1482-1487.

8. Ford E.S. Proportion of the decline in cardiovascular mortality disease due to prevention versus treatment: public health versus clinical care / E. S. Ford, S. Capewell // Annual Review of Public Health. 2011. № 32. P. 5-22.

9. Lozano R., Naghavi M., Foreman K.Global and regional mortality from 235 causes of death for 20 age groups in 1990 and 2010: a systematic analysis for the Global Burden of Disease. Study 2010 // Lancet. 2012. Vol. 380. P. 2095-128.

10. Di Cesare M., Khang Y.H., Asaria P. Inequalities in non-communicable diseases and effective responses // Lancet. 2013. № 381. P. 585-597.

11. Yuanli L., Gonghuan Y., Yixin Z. // The Lancet. Policy dialogue on China's changing burden of disease. 2013. Vol. 381 (9882). P. 1961-1962.

\section{References}

1. Blum David I. The State of Health Care Worldwide // Global Health.12 (2014): 6-11.
2. The Global Plan of Combating Diabetes 20112021. The International Diabetes Federation. Brussels. 2010. P. 7.

3. Monitoring the Risk Factors of Noncommunicable Diseases. The Principle of Phased Implementation Proposed by the WHO. A Brief Review / R. Bonita, M. de Kujerten,T. Duajer i dr. Zheneva: VOZ. 2001.

4. Kontis V., Mathers C.D., Rehm J.Contribution of six risk factors to achieving the $25 \times 25$ non-communicable disease mortality reduction target: a modelling study // The Lancet. 2014. Vol. 384. Pp. 427-437.

5. Di Cesare M., Bennett. J.E., Best N. The contributions of risk factor trends to cardiometabolic mortality decline in 26 industrialized countries // International Journal of Epidemiology. 2013. № 42. P. 838-848.

6. Ezzati M. Riboli E. Behavioral and dietary risk factors for non-communicable diseases // New England Journal of Medicine: Research \& Review. 2013. Vol. 369. Pp. 954-964.

7. Ezzati M., Riboli E. Can non-communicable diseases be prevented? Lessons from studies of populations and individuals // Science. 2012. Vol. 337. P. 1482-1487.

8. Ford E.S. Proportion of the decline in cardiovascular mortality disease due to prevention versus treatment: public health versus clinical care / E. S. Ford, S. Capewell // Annual Review of Public Health. 2011. № 32. P. 5-22.

9. Lozano R., Naghavi M., Foreman K.Global and regional mortality from 235 causes of death for 20 age groups in 1990 and 2010: a systematic analysis for the Global Burden of Disease. Study 2010 // Lancet. 2012. Vol. 380. P. 2095-128.

10. Di Cesare M., Khang Y.H., Asaria P. Inequalities in non-communicable diseases and effective responses // Lancet. 2013. № 381. P. 585-597.

11. Yuanli L., Gonghuan Y., Yixin Z. // The Lancet. Policy dialogue on China's changing burden of disease. 2013. Vol. 381 (9882). P. 1961-1962. 


\section{Приложение 1 \\ Анамнестическая анкета \\ Annex 1 \\ Anamnestic Questionnaire}

Амбулаторная карта №

Дата заполнения

Дата рождения Возраст (полных лет)

Пол

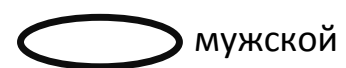

1. Говорил ли Вам врач, что у Вас повышенное артериальное давление?
ДА
HET

2. Говорил ли Вам врач, что у Вас ишемическая болезнь сердца (стенокардия или инфаркт миокарда)? ДА

HET

3. Говорил ли Вам врач, что у Вас цереброваскулярные заболевания (в т.ч. перенесенный инсульт)? ДА HET

4. Говорил ли Вам врач, что у Вас сахарный диабет или повышенный уровень глюкозы (сахара) в крови? ДА HET

5. Говорил ли Вам врач, что у Вас онкологическое заболевание? ДА HET

6. 5. Говорил ли Вам врач, что у Вас туберкулез легких?

ДА HET

7. Был инфаркт миокарда у Ваших близких родственников (матери, родных сестер в возрасте до 65 лет, или у отца, родных братьев) в возрасте до 55 лет?

HET

HE ЗНАЮ

8. Был ли инсульт у Ваших близких родственников (матери, родных сестер в возрасте до 65 лет, или у отца, родных братьев в возрасте до 55 лет)?
ДА
HET
HE ЗНАЮ

9. Был у Ваших близких родственников (матери, родных сестер в возрасте до 65 лет, или у отца, родных братьев в возрасте до 55 лет) сахарный диабет?
ДА
HET
HE ЗНАЮ

10. Был у Ваших близких родственников рак толстой кишки или полипы, возникшие в возрасте до 50 лет?

ДА НЕТ НЕ ЗНАЮ

11 ДЛЯ МУЖЧИН. Просыпаетесь Вы ночью, чтобы помочиться 2 и более раз?

11 ДЛЯ ЖЕНЩИН. Был у Ваших близких родственников (матери, родных сестер, бабушки) рак молочной железы, возникший в возрасте до 50 лет? ДА

HET

12. Возникали ли у Вас, когда Вы поднимаетесь по лестнице, идете в гору, спешите, или выходите из теплого помещения на холодный воздух, боль, чувство давления, жжения в груди или левой половине грудной клетки с распространением в левую руку?

ДА

HET

13. Бывают ли у Вас ежегодно периоды ежедневного кашля с выделением мокроты в течение примерно трех месяцев в году?

$$
\text { ДА HЕT }
$$

14. Бывает ли у Вас кровохарканье?
ДА
HET

15. потеряли Вы вес за последнее время без известных причин (без соблюдения диет, или увеличение физической активности)?
ДА
HET

16. Бывают ли у Вас примеси крови в кале?

$$
\text { ДА НЕT }
$$

17. Курите ли Вы (курение одной и более сигарет в день) или курили в прошлом?

$$
\text { ДА HET }
$$

18. Вы тратите на ходьбу в умеренном или быстром темпе (включая дорогу до места работы и обратно) более 30 минут ежедневно? 

«Научный результат». Серия «Медицина и фармация». - Т.2, №1(7), 2016.

19. Употребляете ли Вы ежедневно около 400 граммов (или 4-5 порций) фруктов и овощей (не считая картофеля)?
ДА
HET

20. Употребляете ли Вы 100 граммов рыбы 2 раза в неделю? ДА

HET

21. Обращали ли Вы внимание на содержание жира и / или холестерина в продуктах при покупке (на этикетках, упаковках) или при приготовлении пищи ДА

HET

22. Есть ли у Вас привычка подсаливать приготовленную пищу, не пробуя ее? ДА

HET

23. Отмечали ли Вы в последнее время увеличение выпадения волос при расчесывании, ломкость ногтей или сухость кожи?
ДА
HET

24. Отмечали ли Вы в последнее время немотивированные приступы сердцебиения или перебоев в работе сердца? ДА

HET

25. Часто ли у Вас возникает плохое настроение, тоска или чувство безнадежности в течении последнего месяца?
ДА
HET

26. Часто замечали Вы отсутствие интереса или удовольствия от вещей, которые обычно Вас заинтересовали, приносили Вам удовольствие, в течении последнего месяца? 